(C) OPEN ACCESS

\title{
Prospective, multicentre study of external ventricular drainage-related infections in the UK and Ireland
}

\author{
Aimun A B Jamjoom, ${ }^{1}$ Alexis J Joannides, ${ }^{2}$ Michael Tin-Chung Poon, ${ }^{1}$ Aswin Chari, ${ }^{3}$ \\ Malik Zaben, ${ }^{4}$ Mutwakil A H Abdulla, ${ }^{2,4}$ Joy Roach, ${ }^{5}$ Laurence J Glancz, ${ }^{6}$ Anna Solth, ${ }^{7}$ \\ John Duddy, ${ }^{8}$ Paul M Brennan, ${ }^{1}$ Roger Bayston, ${ }^{9}$ Diederik 0 Bulters, ${ }^{10}$ \\ Conor L Mallucci, ${ }^{10}$ Michael D Jenkinson, ${ }^{11,12}$ William P Gray, ${ }^{4}$ Jothy Kandasamy, \\ Peter J Hutchinson, ${ }^{2}$ Angelos G Kolias, ${ }^{2}$ Aminul I Ahmed, ${ }^{5}$ on behalf of the British \\ Neurosurgical Trainee Research Collaborative
}

For numbered affiliations see end of article.

\section{Correspondence to} Mr Aimun A B Jamjoom, Department of Clinical Neuroscience, Western General Hospital, Crewe Road South, Edinburgh, EH42XU; aabjamjoom@gmail.com

Received 10 May 2017 Revised 16 September 2017 Accepted 9 October 2017 Published Online First 25 October 2017

\section{SLinked}

- http://dx.doi.org/10.1136/ jnnp-2017-317105

\section{CrossMark}

To cite: Jamjoom $A A B$, Joannides AJ, Poon MT-C, et al. J Neurol Neurosurg Psychiatry 2018;89:120-126.

\section{ABSTRACT}

Objectives External ventricular drain (EVD) insertion is a common neurosurgical procedure. EVD-related infection (ERI) is a major complication that can lead to morbidity and mortality. In this study, we aimed to establish a national ERI rate in the UK and Ireland and determine key factors influencing the infection risk.

Methods A prospective multicentre cohort study of EVD insertions in 21 neurosurgical units was performed over 6 months. The primary outcome measure was 30-day ERI. A Cox regression model was used for multivariate analysis to calculate HR.

Results A total of 495 EVD catheters were inserted into 452 patients with EVDs remaining in situ for 4700 days (median 8 days; IQR 4-13). Of the catheters inserted, 188 (38\%) were antibiotic-impregnated, 161 (32.5\%) were plain and $146(29.5 \%)$ were silver-bearing. A total of 46 ERIs occurred giving an infection risk of $9.3 \%$.

Cox regression analysis demonstrated that factors independently associated with increased infection risk included duration of EVD placement for $\geq 8$ days $(H R=2.47(1.12-5.45) ; p=0.03)$, regular sampling (daily sampling ( $H R=4.73(1.28-17.42), p=0.02)$ and alternate day sampling $(H R=5.28(2.25-12.38) ; p<0.01)$. There was no association between catheter type or tunnelling distance and ERI.

Conclusions In the UK and Ireland, the ERI rate was 9.3\% during the study period. The study demonstrated that EVDs left in situ for $\geq 8$ days and those sampled more frequently were associated with a higher risk of infection. Importantly, the study showed no significant difference in ERI risk between different catheter types.

\section{INTRODUCTION}

The insertion of an external ventricular drain (EVD) is one of the the most common neurosurgical procedures. It was first described by Claude-Nicholas Le Cat in 1744 when he punctured the ventricle and left a wick in situ for congenital hydrocephalus. ${ }^{1}$ Since then, the procedure has seen significant refinement in its technique, an expansion of its indications and technological advances in the materials used for drainage and insertion. ${ }^{2}$ In the last two decades, EVD research has focused on improving the accuracy of ventricular access and infection control. EVD-related infection (ERI) is a significant complication that can lead to increased morbidity and healthcare costs. ${ }^{3}$ ERI has a reported rate in the literature between $1 \%$ and $45 \% .{ }^{4}$ This wide variation is driven by differing definitions of ERI and study methodology. A range of factors are reported to be associated with increased ERI risk including duration of drainage, cerebrospinal fluid (CSF) leak, frequency of sampling and underlying aetiology. ${ }^{4-8}$ Efforts to reduce ERI risk have included the introduction of EVD care bundles, the use of perioperative or continuous prophylactic antibiotics and the development of antimicrobial-impregnated catheters. ${ }^{5-11}$ Observational studies of ERI rates have been primarily retrospective and those which collected data prospectively have been restricted to single neurosurgical units (NSUs). ${ }^{42-16}$ Studies with these limitations have inherent practice and selection biases, do not accurately reflect current national practice for generating health economic models nor do they lend themselves to generating a baseline against which future multicentre randomised trials can be designed and adequately powered. Using prospectively collected data from multiple NSUs, we aimed to determine the national infection rate, assess the incorporation of current evidence into clinical practice and identify parameters associated with increased infection rates that can be interrogated in future clinical studies and be used to target preventative measures.

\section{MATERIALS AND METHODS}

A prospective, multicentre observational study of EVD management and infection rates was conducted across 21 NSUs in the UK and Ireland. Primary data collection took place over 6 months between November 2014 and May 2015 followed by 30 -day follow-up. The inclusion criteria were any tunnelled EVD catheter inserted in a patient of any age without evidence of pre-existing CSF infection. The study protocol was approved by the Society of British Neurological Surgeons (SBNS) Academic Committee and conducted by the British Neurosurgical Trainee Research Collaborative (BNTRC). ${ }^{17}$ Strengthening the Reporting of Observational Studies in Epidemiology 
(STROBE) checklist was used to guide the preparation of this manuscript. ${ }^{18}$

\section{Data collection and outcome measures}

The BNTRC is a network of neurosurgical trainees in the UK and Ireland who conduct multicentre collaborative research with the support of the academic committee of the Society of British Neurological Surgeons. ${ }^{19}{ }^{20}$ Within each NSU, a trainee lead coordinated local patient identification and data collection working closely with a team of collaborators, including a Consultant lead. Data were entered to the Outcome Registry Intervention and Operation Network (ORION) based at the University of Cambridge. ORION is a secure database, which complies with the Department of Health Information Governance policy and meets data security standards of the Information Governance Toolkit of the Health and Social Care Information Centre. The study protocol was approved by the audit and clinical governance committee of each participating hospital.

A range of demographic and operative parameters were captured including age, sex, underlying aetiology, primary surgeon grade, type of catheter (plain, antibiotic-impregnated or silver-bearing), length of tunnelling and frequency of subsequent CSF sampling protocol (daily, alternate days, 1-2 times per week, no sampling and unknown). The primary outcome was ERI within 30 days. A pragmatic definition of ERI was used: evidence of positive CSF culture (and/or gram stain) or clinical suspicion of ERI by managing team due to CSF pleocytosis, elevated serum inflammatory markers and clinical signs including fever, meningism and altered conscious level. ${ }^{21}$ Secondary outcome measures included: mortality in the NSU, functional status at discharge using the modified Rankin Score (mRS) and permanent CSF diversion at 30 days.

\section{Statistical analysis}

The statistical approach follows the principles outlined in the study protocol. ${ }^{17}$ In the univariate analyses, $\chi^{2}$ test or Fisher's exact test was used to compare clinical variables between infected and non-infected cases and outcomes between different types of catheters. The duration of prophylactic antibiotic therapy and time to infection was compared using the Kruskal-Wallis test. In the multivariate analyses of ERIs, prespecified independent variables were entered which included type of catheter, length of tunnelling, CSF sampling frequency and dichotomised duration of EVD use around the median. These were chosen because of their known or hypothesised association with ERI. To examine the rate of ERIs, HRs for ERI were calculated using Cox regression model adjusted for age, sex, type of catheter, length of tunnelling, CSF sampling frequency and dichotomised duration of EVD use. The time-at-risk period was from date of EVD insertion to ERI, death or 30 days after EVD insertion. To examine factors associated with ERIs a multiple logistic regression model using the prespecified independent variables was used. As a sensitivity analysis to test whether NSU influenced the odds of infection within 30 days of EVD insertion, a multilevel mixed effect logistic regression was performed using hospitals as clusters. This model was compared with a single-level multiple logistic regression model using the likelihood ratio test. We used STATA V.13.0 (StataCorp) for conducting statistical tests and generating graphical outputs. A p value of $<0.05$ denoted statistical significance.
Table 1 Demographic data for 495 EVD catheters

\begin{tabular}{|lcl}
\hline Patient characteristics & No. & $\%$ \\
\hline Age & & \\
\hline Mean (SD) & $51.8(19.2)$ & - \\
\hline Median (IQR) & $54(41-67)$ & - \\
\hline Age groups & & \\
\hline $0-17$ & 37 & 7.4 \\
\hline $18-29$ & 34 & 6.8 \\
\hline $30-39$ & 45 & 9.1 \\
\hline $40-49$ & 78 & 15.8 \\
\hline $50-59$ & 113 & 22.8 \\
\hline $60-69$ & 103 & 20.8 \\
\hline $70-79$ & 66 & 13.3 \\
\hline $80-89$ & 15 & 3.0 \\
\hline $90+$ & 4 & 1.0 \\
\hline Gender & & \\
\hline Female & 261 & 52.7 \\
\hline Male & 234 & 47.3 \\
\hline Pathology & & \\
\hline Congenital, IIH and NPH & 12 & 2.4 \\
\hline Shunt dysfunction & 19 & 3.8 \\
\hline Neurovascular & 321 & 64.9 \\
\hline Other & 20 & 4.0 \\
\hline Trauma & 35 & 7.1 \\
\hline Tumour & 88 & 17.8 \\
\hline Preoperative ASA status & 117 & 23.6 \\
\hline 1 & 108 & 21.8 \\
\hline 2 & 111 & 5.7 \\
\hline 3 & 28.5 \\
\hline 4 & & \\
\hline 5 & & \\
\hline ASA, Ameris & & \\
\hline
\end{tabular}

ASA, American Society of Anaesthesiologists; EVD, external ventricular drain; IIH, idiopathic intracranial hypertension; NPH, normal pressure hydrocephalus.

\section{RESULTS}

\section{Demographic and operative data}

During the 6-month period, a total of 495 EVD catheters were inserted in 452 patients. The follow-up was 12308 days (median 30 days; IQR 23-30). The median age at time of insertion was 54 years and $261(52.7 \%)$ catheters were inserted in female patients. Hydrocephalus secondary to a neurovascular aetiology (64.9\%) was the the most common indication for EVD insertion followed by tumours (17.8\%) and trauma (7.1\%). The baseline demographic characteristics of the cohort are presented in table 1.

The majority (98.6\%) of EVDs were inserted in the operating theatre and the remainder (1.4\%) were inserted in the intensive care unit. In most cases (85.3\%) prophylactic systemic antibiotics were given at induction. A small number (3.2\%) had prolonged systemic antibiotic prophylaxis with the remaining groups either already on antibiotics $(3.8 \%)$ or receiving no systemic antimicrobial prophylaxis (7.7\%). Neurosurgical trainees were the primary surgeon in $424(85.7 \%)$ EVD procedures with $66 \%$ of the total being inserted by senior trainees. Of the catheters inserted, 188 (38\%) were antibiotic-impregnated, 161 (32.5\%) were plain and 146 (29.5\%) were silverbearing catheters. EVDs remained in situ for a total of 4700 days (median of 8 days (IQR 4-13)). Table 2 presents operative data regarding the EVD procedures. 


\begin{tabular}{|c|c|c|}
\hline Operative characteristics & No. & $\%$ \\
\hline \multicolumn{3}{|l|}{ Length of tunnelling } \\
\hline $0-5 \mathrm{~cm}$ & 119 & 24.0 \\
\hline $5-10 \mathrm{~cm}$ & 359 & 72.5 \\
\hline$>10 \mathrm{~cm}$ & 17 & 3.4 \\
\hline \multicolumn{3}{|l|}{ Postoperative CT head scan } \\
\hline No & 100 & 20.2 \\
\hline Yes & 395 & 79.8 \\
\hline \multicolumn{3}{|l|}{ CSF sampling frequency } \\
\hline No sampling & 129 & 26.1 \\
\hline $1-2$ times per week & 234 & 47.3 \\
\hline Alternate days & 24 & 4.9 \\
\hline Daily & 7 & 1.4 \\
\hline Unknown & 101 & 20.4 \\
\hline \multicolumn{3}{|l|}{ Catheter type } \\
\hline Plain & 161 & 32.5 \\
\hline Antibiotic-impregnated & 188 & 38 \\
\hline Silver-bearing & 146 & 29.5 \\
\hline \multicolumn{3}{|l|}{ Primary surgeon } \\
\hline Intern/Foundation doctor & 5 & 1.0 \\
\hline Junior neurosurgery trainee & 97 & 19.6 \\
\hline Senior neurosurgery trainee & 327 & 66.1 \\
\hline Consultant/Attending & 66 & 13.3 \\
\hline
\end{tabular}

CSF, cerebrospinal fluid; EVD, external ventricular drain.

\section{Infection outcome}

Infection rate

Forty-six ERIs were observed during the follow-up period. The overall risk of infection was $9.3 \%(n=46 / 495)$ within 30 days. One ERI had a missing date of infection. Of the remaining 45 ERIs, six occurred after EVD removal. Therefore, the rate of ERI while an EVD was in place was $0.8 \%$ per day. The median time to infection was 9 days (IQR 5-15). For 25 cases of ERI, pathogens were isolated with a total of 29 organisms. The most common organisms were coagulase negative staphylococcus $(\mathrm{n}=10,34.5 \%)$, followed by Staphylococcus aureus $(\mathrm{n}=6$, 20.7\%) and Enterococcus spp. $(\mathrm{n}=3,10.3 \%)$. Table 3 includes the frequency of all isolated microbiological organisms.

The median length of treatment with antibiotics was 10 days (IQR 7-14). A comparison of infected versus uninfected cases using the $\mathrm{X}^{2}$ test demonstrated that a significantly higher percentage of infected cases had a catheter in situ for $\geq 8$ days

\begin{tabular}{|c|c|c|}
\hline Cultured organisms & No. & $\%$ \\
\hline Coagulase-negative staphylococcus* & 10 & 34.5 \\
\hline Staphylococcus aureus & 6 & 20.7 \\
\hline Enterococcus spp. & 3 & 10.3 \\
\hline Enterobacter spp. & 2 & 6.9 \\
\hline Klebsiella pneumoniae & 2 & 6.9 \\
\hline Morganella morganii & 1 & 3.4 \\
\hline Escherichia coli & 1 & 3.4 \\
\hline Klebsiella sp. & 1 & 3.4 \\
\hline Pseudomonas aeruginosa & 1 & 3.4 \\
\hline Corynebacterium sp. & 1 & 3.4 \\
\hline Streptococcus sp. & 1 & 3.4 \\
\hline
\end{tabular}

\footnotetext{
*Three cases due to Staphylococcus epidermidis.

CSF, cerebrospinal fluid.
}

\begin{tabular}{|c|c|c|c|}
\hline & \multicolumn{2}{|c|}{ Infection within 30 days } & \multirow[b]{2}{*}{$\mathrm{p}$ Value } \\
\hline & No (\%) & Yes (\%) & \\
\hline \multicolumn{4}{|l|}{ Catheter type } \\
\hline Plain & $149(92.5)$ & $12(7.5)$ & 0.314 \\
\hline Antibiotic-impregnated & $174(92.6)$ & $14(7.4)$ & \\
\hline Silver-bearing & $126(86.3)$ & $20(13.7)$ & \\
\hline \multicolumn{4}{|l|}{ CSF sampling frequency } \\
\hline No sampling & $129(100)$ & $0(0)$ & $<0.01$ \\
\hline $1-2$ times per week & $211(90.2)$ & $23(9.8)$ & \\
\hline Alternate days & $15(62.5)$ & $9(26.5)$ & \\
\hline Daily & $3(42.9)$ & $4(57.1)$ & \\
\hline Unknown & $91(90.1)$ & $10(9.9)$ & \\
\hline \multicolumn{4}{|l|}{ Length of tunnelling } \\
\hline $0-5 \mathrm{~cm}$ & $106(89.1)$ & $13(10.9)$ & 0.71 \\
\hline $5-10 \mathrm{~cm}$ & $328(91.4)$ & $31(8.6)$ & \\
\hline$>10 \mathrm{~cm}$ & $15(88.2)$ & $2(11.8)$ & \\
\hline \multicolumn{4}{|l|}{ Primary surgeon } \\
\hline Intern/Foundation doctor & $4(80.0)$ & $1(20.0)$ & 0.25 \\
\hline Junior neurosurgery trainee & $87(89.7)$ & $10(10.3)$ & \\
\hline Senior neurosurgery trainee & $294(89.9)$ & $33(10.1)$ & \\
\hline Consultant & $64(97.0)$ & $2(3.0)$ & \\
\hline \multicolumn{4}{|l|}{ Duration of EVD use* } \\
\hline $0-7$ days & $236(95.9)$ & $10(4.1)$ & $<0.01$ \\
\hline 8+days & $212(85.8)$ & $35(14.2)$ & \\
\hline \multicolumn{4}{|l|}{ CSF diversion } \\
\hline Permanent CSF diversion & $71(91.0)$ & $7(9.0)$ & 0.91 \\
\hline No permanent diversion & $378(90.6)$ & $39(9.4)$ & \\
\hline
\end{tabular}

Comparisons made using $\mathrm{X}^{2}$ test.

*Two cases excluded as no valid exit date.

CSF, cerebrospinal fluid; EVD, external ventricular drain.

$(77.8 \%)$ compared with uninfected cases $(47.3 \%)(p<0.01)$. Similarly, a significantly higher percentage of infected cases had their EVD sampled more frequently (daily or on alternate days) compared with uninfected cases $(p<0.01)$. Conversely, there was no significant difference between infected and uninfected cases with regard to the different catheter types $(p=0.31)$, length of tunnelling $(p=0.71)$ or the likelihood of permanent CSF diversion at 30 days $(p=0.91)$. Table 4 provides comparison between the infected and uninfected cases.

\section{Comparison between EVD catheter types infection outcome}

A $\mathrm{X}^{2}$ test for homogeneity showed there was no significant difference between the distribution of catheter types across the cohort age groups, gender and underlying pathology. A comparison of the infection rate according to catheter types showed that silver-bearing catheters had the highest infection rate $(13.7 \%)$, compared with plain $(7.5 \%)$ and antibiotic-impregnated catheters (7.4\%), but this did not reach statistical significance $(p=0.09)$. The median time to infection was longest for the antibiotic-impregnated catheters (11 days) followed by plain catheters (8 days) and then silver-bearing (7 days). Infections on a background of antibiotic-impregnated catheters received antibiotic treatment for a median of 14 days, silver-bearing catheters received a median of 10 days' therapy and plain catheters received 7.5 days. Table 5 provides a summary of clinical and infection variation between the catheter subtypes. 
Table 5 Comparison of clinical and infection parameters between catheter subtypes

\begin{tabular}{|c|c|c|c|c|c|c|c|}
\hline & \multicolumn{2}{|l|}{$\begin{array}{l}\text { Plain } \\
(n=161)\end{array}$} & \multicolumn{2}{|c|}{ Antibiotic-impregnated $(n=188)$} & \multicolumn{2}{|c|}{$\begin{array}{l}\text { Silver-bearing } \\
(n=146)\end{array}$} & \multirow[b]{2}{*}{$\mathrm{p}$ Value } \\
\hline & No. & $\%$ & No. & $\%$ & No. & $\%$ & \\
\hline \multicolumn{8}{|l|}{ 30-day infection } \\
\hline No & 149 & 92.6 & 174 & 92.6 & 126 & 86.3 & 0.09 \\
\hline Yes & 12 & 7.5 & 14 & 7.4 & 20 & 13.7 & \\
\hline \multicolumn{8}{|l|}{ Time to infection (median) } \\
\hline & $8(1-29)$ & - & $11(2-29)$ & - & $7(1-39)$ & - & 0.19 \\
\hline \multicolumn{8}{|l|}{ Length of antibiotic therapy for ERI (median) } \\
\hline & $7.5(1-28)$ & - & $14(1-87)$ & - & $10(1-37)$ & - & 0.23 \\
\hline \multicolumn{8}{|l|}{ Cultured microorganisms } \\
\hline Coagulase-negative staphylococcus (p) & 4 & 36.3 & 2 & 25.0 & 4 & 40.0 & - \\
\hline Staphylococcus aureus (p) & 3 & 27.3 & 2 & 25.0 & 1 & 10.0 & - \\
\hline Enterococcus spp. (p) & 1 & 9.1 & 2 & 25.0 & - & - & - \\
\hline Enterobacter spp. (n) & - & - & 1 & 12.5 & 1 & 10.0 & - \\
\hline Klebsiella pneumoniae (n) & 1 & 9.1 & - & - & 1 & 10.0 & - \\
\hline Morganella morganii (n) & - & - & - & - & 1 & 10.0 & - \\
\hline Escherichia coli (n) & 1 & 9.1 & - & - & - & - & - \\
\hline Klebsiella (n) & 1 & 9.1 & - & - & - & - & - \\
\hline Pseudomonas aeruginosa (n) & - & - & - & - & 1 & 10.0 & - \\
\hline Corynebacterium (p) & - & - & 1 & 12.5 & - & - & - \\
\hline Streptococcus spp. (p) & - & - & - & - & 1 & 10.0 & - \\
\hline \multicolumn{8}{|l|}{ 30-day mortality* } \\
\hline No & 100 & 70.4 & 145 & 81.0 & 93 & 71.0 & 0.05 \\
\hline Yes & 42 & 29.6 & 34 & 19.0 & 38 & 29.0 & \\
\hline \multicolumn{8}{|l|}{ 30-day mRS* } \\
\hline Good (mRS 0-2) & 50 & 35.2 & 79 & 44.1 & 35 & 26.7 & 0.01 \\
\hline Poor (mRS 3-5) & 50 & 35.2 & 66 & 36.9 & 58 & 44.3 & \\
\hline Death & 42 & 29.6 & 34 & 19.0 & 38 & 29.0 & \\
\hline
\end{tabular}

*30-day mortality and 30-day mRS based on 452 non-duplicate patients; $(p)=$ gram stain positive; $(n)=$ gram stain negative.

ERI, external ventricular drain-related infection; mRS, modified Rankin Score.

Clinical variables associated with infection

There was a significant increase in risk of infection for both daily and alternate day sampling regimens compared with catheters that were sampled 1-2 times per week. Similarly, catheters left in situ for $>8$ days had a higher risk of ERI compared with those removed at 7 days or less ( $\mathrm{OR}=2.54$ (95\% CI 1.14 to 5.7$) ; \mathrm{p}=0.02)$. There was no association between the underlying pathology and the risk of infection in univariate analysis $(\mathrm{p}=0.76)$. Catheters tunnelled $>5 \mathrm{~cm}$ had an ERI OR of 0.75 ( $(95 \% \mathrm{CI} 0.3$ to 1.45$) ; \mathrm{p}=0.29)$ compared with those with shorter tunnelling. Nelson-Aalen cumulative hazard estimate curves of catheter type, EVD duration and length of tunnelling are shown in figure 1. To determine whether NSU had an effect on ERI, a likelihood ratio test, comparing a multilevel mixed effects logistic regression using hospitals as clusters, was performed; ERI rate did not significantly vary between hospitals $(\mathrm{p}=0.16)$. A Cox regression model adjusted for age, sex, type of catheter, length of tunnelling, CSF sampling frequency and duration of EVD use based on 441 EVDs where time-at-risk was available identified a higher rate of infection for catheters kept in situ for 8 days or longer ( $\mathrm{HR}=2.47$ (95\% CI 1.12 to 5.45$)$; $\mathrm{p}=0.03$ ). Using a similar analysis, there was no significant difference in ERI risk between catheter types (table 6).

\section{Paediatric subanalysis}

Among the 37 catheters inserted into patients aged $<18$, there were three infections giving the paediatric cohort an infection rate of $8.1 \%$. The distribution of pathologies between adult and paediatric cases is shown in table 7 . There was a significant $(p<0.01)$ difference in the pathological aetiology necessitating an EVD insertion between the different age groups. Similarly, there was a significant difference of the catheter types inserted into the two cohorts. Particularly, antibiotic-impregnated catheters were preferred in the paediatric cohort constituting $62.2 \%$ of catheters compared with only $36 \%$ of catheters in adults. Importantly, exclusion of the paediatric subcohort did not cause a change in the observed association trends in the Cox regression analysis. Catheter drainage $>8$ days and sampling frequency (daily and alternate days) remained significantly associated with increased ERI risk.

\section{Mortality and functional outcome}

There were 114 (25.2\%) deaths observed within 30 days after EVD insertion in the 452 patients recruited in our study (table 5). Of the surviving patients, $164(48.5 \%)$ had a good functional outcome (mRS $0-2)$ and $174(51.5 \%)$ had a poor outcome (mRS $3-4)$.

\section{DISCUSSION}

Using a network of neurosurgical trainees and the BNTRC infrastructure, we were able to capture data on a national level and provide the largest prospective multicentre observational study of EVD management and infection rate in the literature. We confirmed 46 ERIs from 495 catheters giving an infection risk of 9.3\% (or 9.8 ERIs per 1000 catheter days). This falls 
A.

NA curve of EVD infection rate by type of EVD catheter

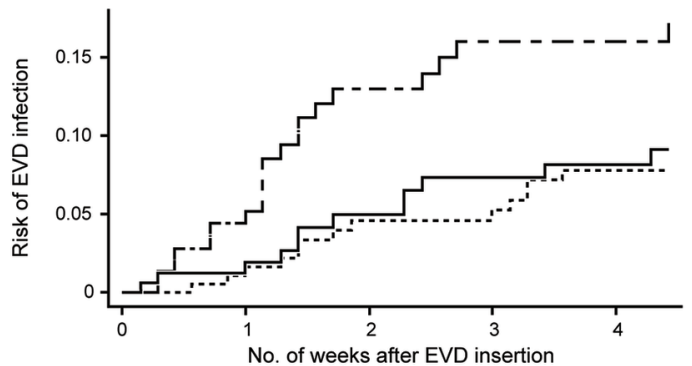

Number at risk

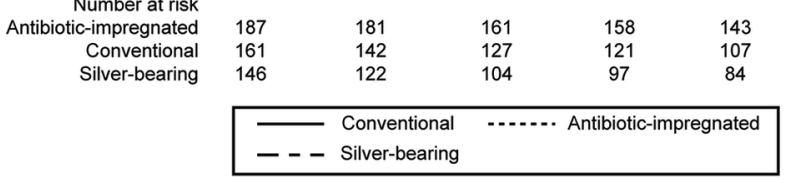

B.

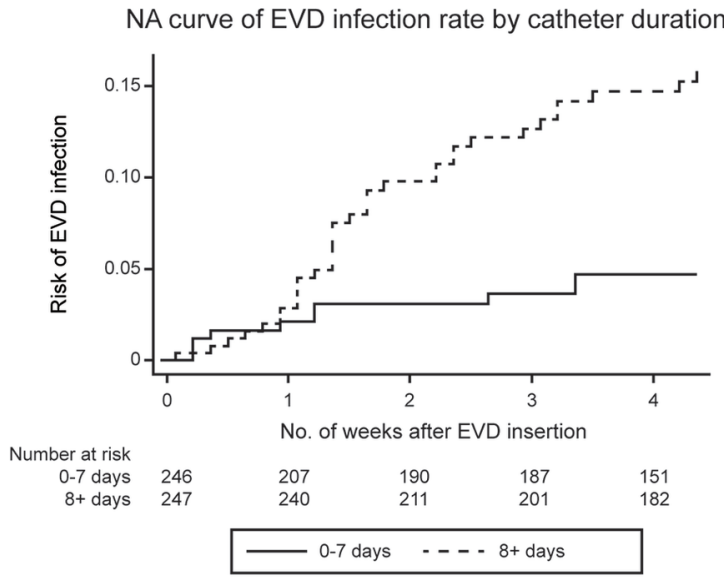

C.

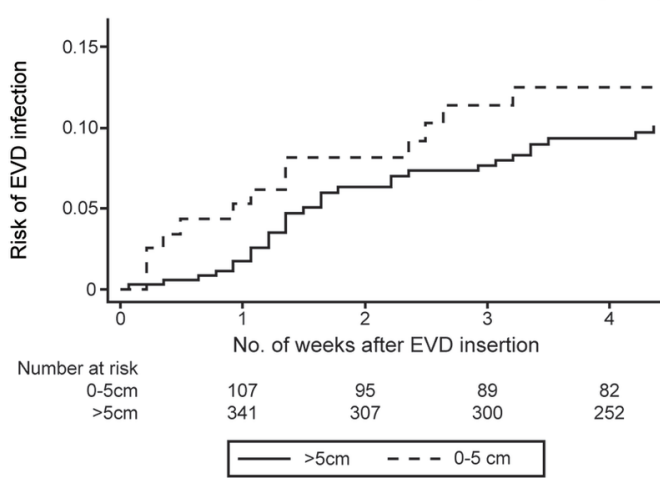

Figure 1 Nelson-Aalen cumulative hazard estimate of EVD infection by (A) type of EVD catheter: log-rank test of equality showed $p=0.06$, (B) catheter duration: log-rank test of equality showed $p<0.01$, (C) tunnelling length: log-rank test of equality showed $p=0.41$. EVD, external ventricular drain.

within the wide range of quoted infection risk within the literature; however, direct comparison is limited due to the differing definitions of ERIs used. We chose a pragmatic definition which included culture-positive CSF or other features of ERI (CSF pleocytosis, raised inflammatory markers, clinical features of meningitis) that prompted treatment. This definition is used in a range of studies including a previously published randomised trial assessing the effectiveness of silver-bearing catheters. ${ }^{21} \mathrm{~A}$

\begin{tabular}{|c|c|c|c|}
\hline & HR & $95 \% \mathrm{Cl}$ & $\mathrm{p}$ Value \\
\hline \multicolumn{4}{|l|}{ Catheter type } \\
\hline Plain & Ref & - & - \\
\hline Antibiotic-impregnated & 0.87 & 0.37 to 2.03 & 0.75 \\
\hline Silver-bearing & 1.35 & 0.63 to 2.88 & 0.44 \\
\hline \multicolumn{4}{|l|}{ Length of tunnelling } \\
\hline $0-5 \mathrm{~cm}$ & Ref & - & - \\
\hline$>5 \mathrm{~cm}$ & 0.75 & 0.36 to 1.55 & 0.44 \\
\hline \multicolumn{4}{|l|}{ CSF sampling frequency } \\
\hline 1-2 times per week & Ref & - & - \\
\hline Alternate days & 5.28 & 2.25 to 12.38 & $<0.01$ \\
\hline Daily & 4.73 & 1.28 to 17.42 & 0.02 \\
\hline Unknown & 1.26 & 0.56 to 2.82 & 0.58 \\
\hline \multicolumn{4}{|l|}{ Duration of EVD use } \\
\hline $0-7$ days & Ref & - & - \\
\hline$\geq 8$ days & 2.47 & 1.12 to 5.45 & 0.03 \\
\hline
\end{tabular}

${ }^{*}$ Cox regression model adjusted for age and sex based on 441 EVDs where date of exit (infection, death or 30 days) was available.

CSF, cerebrospinal fluid; EVD, external ventricular drain.

meta-analysis by Ramanan and colleagues looked at ERIs and found a total of 35 observational studies reported an overall infection rate of 11.4 ERIs per 1000 catheter days. ${ }^{4}$ Within their study, the authors found several definitions of infection rates used by the 35 studies and the pragmatic definition used in our study was the second most used definition (seven studies). The meta-analysis of these seven studies found a total of 17 ERIs per 1000 catheter days (95\% CI 10 to 24.1 ). This value was double our findings of 9.8 ERIs per 1000 catheter days. This comparison, despite its limitations, reflects that the UK and Ireland's ERI rate is consistent with the literature and may be lower than studies with an equivalent ERI definition.

One of our key findings was an increased ERI rate with a longer duration of EVD placement. Catheters in situ for $\geq 8$ days were associated with a greater than twofold increase in rate of developing an ERI compared with those in place for 7 days or less. The literature has conflicting evidence on the relationship between the length of EVD placement and ERI. Some studies have shown no association with duration of EVD placement and ERI, ${ }^{22}$ while others corroborated our findings. ${ }^{7} 8$ Moreover, some authors have recommended routine replacement of EVDs, ${ }^{23}$ while others argue against this. ${ }^{6}$ Wong and colleagues interrogated this question in a small RCT where they compared

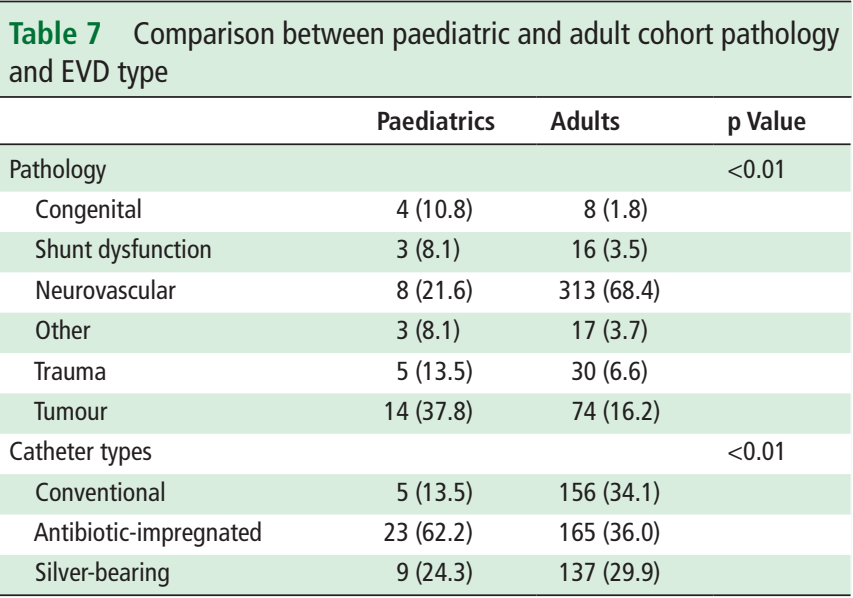

EVD, external ventricular drain 
routine EVD change (every 5 days) to no change. ${ }^{24}$ The study found no difference in ERIs but a trend to higher infection in the routine change group; the authors advised against the use of regular pre-emptive EVD replacement. Our data also showed that an increased frequency of CSF sampling was found to be a significant parameter associated with ERI risk. In keeping with this, several studies have found that patients with an ERI were sampled more frequently than those without an ERI. ${ }^{6} 7$ Their statistical approaches may have been biased by the fact that the number of CSF samples increased with the duration of EVD placement. Our approach did not use absolute numbers of samples taken but rather the sampling frequency protocol. Though this approach has its benefits, we are unable to conclude a direct causal relationship between sampling frequency and ERI. When assessed in the Cox regression model, we found that both catheter duration and sampling frequency were associated with a significantly higher ERI risk. Based on the literature and our data, we conclude that catheters should be removed as early as possible and a lower frequency sampling protocol should be considered as this may help reduce ERI risk.

The development of antimicrobial impregnated catheters (both antibiotic-bearing and silver-bearing catheters) has been a major approach for combating ERI risk. The most commonly used antibiotic-impregnated catheters contain clindamycin and rifampicin, while the silver-bearing catheters contain silver nanoparticles. These catheters impede and kill bacteria with the goal of reducing ERI risk and several studies have attempted to assess their effectiveness. Despite heterogeneity, meta-analyses of these data have broadly pointed towards the effectiveness of impregnated catheters compared with plain catheters. ${ }^{9}{ }^{10}$ Interestingly, our study showed that despite this body of evidence, almost a third of catheters inserted in the UK and Ireland were plain catheters. Our study also failed to demonstrate a significant difference in infection risk between the three different catheter types after adjusting for a range of clinical variables, although there was a trend towards silver-bearing catheters having the highest ERI rate. This is in contrast to a previous trial that suggest silver-bearing catheters are more effective than plain. ${ }^{21}$ In meta-analyses, antibiotic-impregnated catheters have shown more pronounced reductions in ERI risk compared with plain catheters when compared with reductions of risk seen in silver versus plain catheters. ${ }^{9} 10$ This reflects evidence from central venous catheters that found superiority of antibiotic-impregnated catheters compared with those coated with silver in reducing bloodstream infections. ${ }^{25}$ In the UK and Ireland, a major multicentre RCT (The British Antibiotic and Silver Impregnated Catheters for ventriculoperitoneal Shunts trial; BASICS trial) is currently under way aiming to assess the comparative efficacy of antibiotic-bearing, silver-bearing and plain catheters in reducing CSF infections for patients with a ventriculoperitoneal shunt. ${ }^{26}$ The results of this trial will be of great interest to the field and help guide future trial designs looking at a similar question in EVD care. While our data do not support the routine use of antibiotic-bearing and silver-bearing EVD catheters to reduce ERI risk, it highlights an important cost implication that warrants further study since antibiotic-bearing and silver-bearing catheters are substantially more expensive.

In our study, the majority of cultured organisms were gram-positive $(68.9 \%)$ and staphylococci were the most common agent (55.1\%). The predominance of staphylococci mirrors other findings in the literature. ${ }^{7827}$ Most cultured organisms from all three catheter types were gram-positive organisms: plain catheters (63.6\%), antibiotic-impregnated catheters $(87.5 \%)$ and silver-bearing catheters $(60 \%)$. These findings run counter to trends described in the literature of a shift towards gram-negative organisms. ${ }^{61228}$ A range of explanations has been proposed including the impact of antimicrobial-impregnated catheters. In vitro investigation of silver-bearing catheters found a greater eradication of Staphylococcus epidermidis compared with Escherichia coli. ${ }^{29}$ It is of interest that in our data the two cases of Enterobacter were in patients with antibiotic-impregnated and silver-bearing catheters. Atkinson and colleagues concluded that their observed increase in gram-negative organisms was related to the use of silver-bearing catheters; however, their small sample placed limitations on this conclusion. ${ }^{6}$ Prolonged systemic prophylactic antibiotics during drainage has also been implicated in a shift towards more gram-negative microorganisms. $^{28}{ }^{30}$ In our study, only a small percentage $(3.2 \%)$ of cases received prolonged systemic antibiotics which may, in part, explain the dominance of gram-positive organisms.

This study has a number of limitations. First, we were unable to confirm case ascertainment in individual NSUs. In part, this was due to the nature of rotational neurosurgical training which meant trainees would move units, not allowing for continuous data collection in some units. This may have led to an under-reporting of inserted EVDs which could have led to bias in the results. Second, our choice of ERI definition could be open to criticism as it places an onus on the managing clinician to determine the presence of ERIs in cases without a positive CSF culture. This may overestimate the number of ERIs in our cohort due to the presence of false positives. However, as argued above, we believe that the definition is pragmatic, holds relevance for day-to-day clinical practice and has been used in previous RCTs. ${ }^{21}$ Third, NSUs may have different protocols for EVD management that influence the risk of ERIs. In particular, a strict infection control protocol for manipulation of EVD is shown to be associated with a lower risk of ERIs. ${ }^{31} 32$ Local EVD management protocols may confound the results shown in our study. This confounding effect is not likely to have had a large impact on our results since our sensitivity analysis did not show a significant difference in local infection rates, but this aspect of EVD care should be taken into account when interpreting the results. Coupled to this, we did not capture data on concomitant infections which may act as a confounder. Within the data, we also found a significant difference in mortality between catheter subtypes. Though an interesting finding, our study was not designed to examine this question and caution should be taken in drawing conclusions around this observation which is at risk of confounders. Finally, many NSUs favour a single catheter type for EVD insertion. Therefore, factors specific to individual units which contribute to infection rate may confound the overall infection risk difference between the three catheter subtypes. While we did not demonstrate a significant difference between the catheter subtypes, the ERIs between the three groups allow us to perform adequate power calculations for future randomised trials.

\section{CONCLUSION}

This study demonstrated an ERI rate of $9.3 \%$ in 21 centres in the UK and Ireland with a predominance of gram-positive organisms. Patients with an EVD left in situ for $\geq 8$ days and who underwent more frequent sampling had a higher risk of infection. Importantly, there was wide variation in the choice of catheter across the country with no significant difference in ERI risk between the types. These findings support the need for a prospective clinical trial to assess the comparative effectiveness of EVD catheter type on ERI risk. 


\section{Author affiliations}

${ }^{1}$ Department of Clinical Neuroscience, Western General Hospital, Edinburgh, UK ${ }^{2}$ Division of Neurosurgery, Department of Clinical Neurosciences, University of Cambridge and Addenbrooke's Hospital, Cambridge, UK

${ }^{3}$ Department of Neurosurgery, Royal London Hospital, London, UK ${ }^{4}$ Department of Neurosurgery, University Hospital of Wales, Cardiff, UK ${ }^{5}$ Wessex Neurological Centre, University Hospitals Southampton, Southampton, UK ${ }^{6}$ Department of Neurosurgery, Queen's Medical Centre, Nottingham, UK ${ }^{7}$ Department of Neurosurgery, Royal Victoria Infirmary, Newcastle, UK ${ }^{8}$ Department of Neurosurgery, Beaumont Hospital, Dublin, Ireland

${ }^{9}$ Division of Rheumatology, Orthopaedics and Dermatology, The University of Nottingham, Nottingham, UK

${ }^{10}$ Department of Neurosurgery, Alder Hey Children's NHS Trust, Liverpool, UK

${ }^{11}$ Department of Neurosurgery, The Walton Centre, Liverpool, UK

${ }^{12}$ Institute of Translational Medicine, University of Liverpool, Liverpool, UK

Contributors All authors have made substantial contributions to conception and design, acquisition of data or analysis and interpretation of data; drafting of the article or revising it critically for important intellectual content and provided final approval of the version to be published. Roles were as follows: AABJ, AIA: study idea conception, protocol development, research infrastructure organisation, data collection, data analysis, manuscript writing/drafting. AJJ: study idea conception, protocol development, online database development/management, manuscript writing/drafting. MT-CP: data analysis (principle), manuscript writing/drafting. $A C, R B, D O B, C L M, M D J, W P G$, JK: protocol development, research infrastructure organisation, manuscript writing/drafting. MZ, PMB: protocol development, research infrastructure organisation, data collection, manuscript writing/drafting. MAHA: research infrastructure organisation, data collection, manuscript writing. JR, LJG, AS, JD: research infrastructure organisation, data collection, manuscript writing/drafting. PJH: study idea conception, protocol development, manuscript writing/drafting. AGK study idea conception, protocol development, research infrastructure organisation, data collection, manuscript writing/drafting.

Funding PJH is supported by a NIHR Research Professorship and the Cambridge NIHR BRC. The study in this report was funded by the Society of British Neurological Surgeons. AABJ is undertaking a PhD funded by the Wellcome Trust. AIA is funded by the Academy of Medical Sciences Clinical Lecturer Starter Grant.

Competing interests None declared.

Provenance and peer review Not commissioned; externally peer reviewed.

Open Access This is an Open Access article distributed in accordance with the terms of the Creative Commons Attribution (CC BY 4.0) license, which permits others to distribute, remix, adapt and build upon this work, for commercial use, provided the original work is properly cited. See: http://creativecommons.org/ licenses/by/4.0/

(c) Article author(s) (or their employer(s) unless otherwise stated in the text of the article) 2018. All rights reserved. No commercial use is permitted unless otherwise expressly granted.

\section{REFERENCES}

1 Kompanje EJ, Delwel EJ. The first description of a device for repeated external ventricular drainage in the treatment of congenital hydrocephalus, invented in 1744 by Claude-Nicolas Le Cat. Pediatr Neurosurg 2003;39:10-13.

2 Srinivasan VM, O'Neill BR, Jho D, et al. The history of external ventricular drainage. J Neurosurg 2014;120:228-36.

3 Beer R, Lackner P, Pfausler B, et al. Nosocomial ventriculitis and meningitis in neurocritical care patients. J Neurol 2008;255:1617-24.

4 Ramanan M, Lipman J, Shorr A, et al. A meta-analysis of ventriculostomy-associated cerebrospinal fluid infections. BMC Infect Dis 2015;15:3.

5 Chatzi M, Karvouniaris M, Makris D, et al. Bundle of measures for external cerebral ventricular drainage-associated ventriculitis. Crit Care Med 2014;42:66-73.

6 Atkinson R, Fikrey L, Jones A, et al. Cerebrospinal fluid infection associated with silverimpregnated external ventricular drain catheters. World Neurosurg 2016;89:505-9.

7 Hoefnagel D, Dammers R, Ter Laak-Poort MP, et al. Risk factors for infections related to external ventricular drainage. Acta Neurochir 2008;150:209-14.
$8 \mathrm{Kim} \mathrm{JH}$, Desai NS, Ricci J, et al. Factors contributing to ventriculostomy infection. World Neurosurg 2012;77:135-40.

9 Cui Z, Wang B, Zhong Z, et al. Impact of antibiotic- and silver-impregnated external ventricular drains on the risk of infections: A systematic review and meta-analysis. $A m$ J Infect Control 2015;43:e23-e32.

10 Sonabend AM, Korenfeld Y, Crisman C, et al. Prevention of ventriculostomy-related infections with prophylactic antibiotics and antibiotic-coated external ventricular drains: a systematic review. Neurosurgery 2011;68:996-1005.

11 Murphy RK, Liu B, Srinath A, et al. No additional protection against ventriculitis with prolonged systemic antibiotic prophylaxis for patients treated with antibiotic-coated external ventricular drains. J Neurosurg 2015;122:1120-6.

12 Camacho EF, Boszczowski I, Basso M, et al. Infection rate and risk factors associated with infections related to external ventricular drain. Infection 2011;39:47-51.

13 Lundberg F, Wady L, Söderström S, et al. External ventricular drainage catheters: effect of surface heparinization on bacterial colonization and infection. Acta Neurochir 2000;142:1377-83.

14 Schultz M, Moore K, Foote AW. Bacterial ventriculitis and duration of ventriculostomy catheter insertion. J Neurosci Nurs 1993;25:158-64.

15 Scheithauer S, Bürgel U, Ryang YM, et al. Prospective surveillance of drain associated meningitis/ventriculitis in a neurosurgery and neurological intensive care unit. J Neurol Neurosurg Psychiatry 2009;80:1381-5.

16 Scheithauer S, Bürgel U, Bickenbach J, et al. External ventricular and lumbar drainageassociated meningoventriculitis: prospective analysis of time-dependent infection rates and risk factor analysis. Infection 2010;38:205-9.

17 British Neurosurgical Trainee Research Collaborative. Prospective Multicentre Audit of External Ventricular Drainage Management and Infection Rates in the United Kingdom and Ireland: National Audit Protocol. 2014. Epub ahead of print https://m edia.wix.com/ugd/ff5354_e7445c68abc3426f92c37621abbc1e56.pdf

18 Vandenbroucke JP, von Elm E, Altman DG, et al. Strengthening the Reporting of Observational Studies in Epidemiology (STROBE): explanation and elaboration. PLOS Med 2007;4:e297.

19 Jamjoom AAB, Kolias AG, Zaben M, et al. External ventricular drainage: Is it time to look at national practice? Br J Neurosurg 2014;2:1.

20 Kolias AG, Cowie CJ, Tarnaris A, et al. Proposal for a British neurosurgical trainee research collaborative. Br J Neurosurg 2012;26:434-5.

21 Har Keong NC, Bulters DO, Richards HK, et al. The SILVER (Silver Impregnated Line Versus EVD Randomized Trial). Neurosurgery 2012;71:394-404.

22 Pfisterer W, Mühlbauer M, Czech T, et al. Early diagnosis of external ventricular drainage infection: results of a prospective study. J Neurol Neurosurg Psychiatry 2003;74:929-32 http://www.ncbi.nlm.nih.gov/pubmed/12810782.

23 Study APE, Mayhall CG, Archer NH, et al. Ventriculostomy-Related Infections. 2010 http://dx.doi.org/101056/NEJM198403013100903

24 Wong GK, Poon WS, Wai S, et al. Failure of regular external ventricular drain exchange to reduce cerebrospinal fluid infection: result of a randomised controlled trial. J Neurol Neurosurg Psychiatry 2002;73:759-61.

25 Gilbert RE, Harden M. Effectiveness of impregnated central venous catheters for catheter related blood stream infection: a systematic review. Curr Opin Infect Dis 2008:21:235-45.

26 Jenkinson MD, Gamble C, Hartley JC, et al. The British antibiotic and silverimpregnated catheters for ventriculoperitoneal shunts multi-centre randomised controlled trial (the BASICS trial): study protocol. Trials 2014;15:4.

27 Lemcke J, Depner F, Meier U. The Impact of Silver Nanoparticle-Coated and AntibioticImpregnated External Ventricular Drainage Catheters on the Risk of Infections: A Clinical Comparison of 95 Patients: Acta neurochirurgica Supplement, 2012:347-50.

28 Lyke KE, Obasanjo 00, Williams MA, et al. Ventriculitis complicating use of intraventricular catheters in adult neurosurgical patients. Clin Infect Dis 2001;33:2028-33.

29 Bayston R, Vera L, Mills A, et al. In vitro antimicrobial activity of silver-processed catheters for neurosurgery. J Antimicrob Chemother 2010;65:258-65.

30 Poon WS, Ng S, Wai S. CSF antibiotic prophylaxis for neurosurgical patients with ventriculostomy: a randomised study. Acta Neurochir Supp/ 1998;71:146 http://www. ncbi.n/m.nih.gov/pubmed/9779169.

31 Flint AC, Rao VA, Renda NC, et al. A simple protocol to prevent external ventricular drain infections. Neurosurgery 2013;72:993-9.

32 Dasic D, Hanna SJ, Bojanic S, et al. External ventricular drain infection: the effect of a strict protocol on infection rates and a review of the literature. $\mathrm{Br} J$ Neurosurg 2006;20:296-300. 